\title{
Designing IBC cells with FFE: long range effects with circuit simulation
}

\author{
Antonius R. Burgers, Ilkay Cesar, Nicolas Guillevin, Agnes A. Mewe, Pierpaolo Spinelli \\ Arthur W. Weeber
}

\begin{abstract}
IBC cells with Front Floating Emitter (FFE) pose different design challenges compared to more conventional IBC cells with FSF (Front Surface Field). The FFE enables hole transport over distances that are large compared to the typical BSF or emitter width.

The core of the cell design is commonly a device simulation in which, because of the computer resources involved, typically one simulates an as small as possible, but representative part of the solar cell. In an IBC cell this corresponds to $1 / 2$ of the BSF and $1 / 2$ of the emitter. Such a unit cell does not account for important geometric features, such as busbars and pads, edges or interruptions in metallization fingers.

We show how to construct an equivalent circuit for our Mercury FFE IBC cells to model features beyond the unit cell efficiently, taking into account the lateral hole transport in the FFE. We compare and calibrate the circuit model against device simulations with quokka.

Index terms - photovoltaic cells, computer simulation, back contact, silicon
\end{abstract}

\section{INTRODUCTION}

In a consortium of Dutch industrial companies, ECN develops an integral solar cell and module concept based on IBC (Interdigitated Back Contact) cell design with front floating emitter design. Although IBC solar cells have been shown to yield very high conversion efficiencies [1], cost effective production of these devices poses challenges.

Full area 6" screen printed mercury IBC cells have been made using a process based upon ECN's n-pasha screen printed process for n-PERT cells [6] and reported in several of our papers. The highest efficiency achieved to date is $21.1 \%$ with an short-circuit current of $41.3 \mathrm{~mA} / \mathrm{cm}^{2}$ (in-house measurement, mismatch corrected, not independently confirmed) illustrating the effectiveness of the FFE passivation.

The consortium targets low cost and high performance, with 6 inch solar cells of high efficiency, aiming at $22-23 \%$ in 2016 .

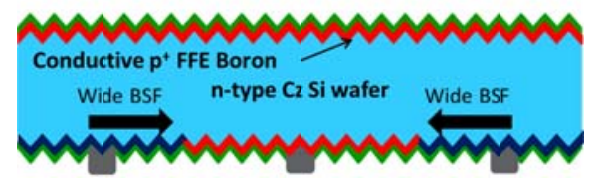

Fig. 1. Cross-section of the Mercury cell; a bifacial n-IBC solar cell with screen-printed contacts.

To allow all contacts to be applied to the rear of the cell, the rear collecting junction (the emitter) is interrupted by a noncollecting junction (the BSF) as illustrated in Fig. 1.
Therefore, any carrier that is photo-generated above a BSF area needs to travel laterally to an emitter area. If the BSF regions become too wide, the collection probability of carriers generated above the BSF will decrease: an effect referred to as electrical shading [2]. To prevent loss in cell performance due to electrical shading for an FSF IBC cell, the typical width of the BSF is in the order of $0.2-0.4 \mathrm{~mm}$ out of a typical cell pitch of $1.5 \mathrm{~mm}$.

The inequality of BSF and emitter widths results in strict patterning tolerances for processing but also has implications for the metallization [3]. For instance for PVD metallization the BSF fingers will be narrow compared to the emitter fingers. Either the metallization has to be thick to achieve sufficient conductivity also on the BSF, or the BSF metallization has to be partially deposited on the emitter, making sure there is sufficient isolation between BSF metallization and emitter. Equal widths of both polarities would allow one to metallize the IBC cell with blanket metallization technologies such as PVD and plating without the need of an isolation layer. This is a significant opportunity for process simplification and cost reduction. To allow for wider BSF regions we have introduced the Mercury IBC cell [3]: an IBC cell with a conductive Front Floating Emitter (FFE). In fact back contact FFE cells have been proposed before, e.g. in $[4,5]$ but have not become a main stream technology.
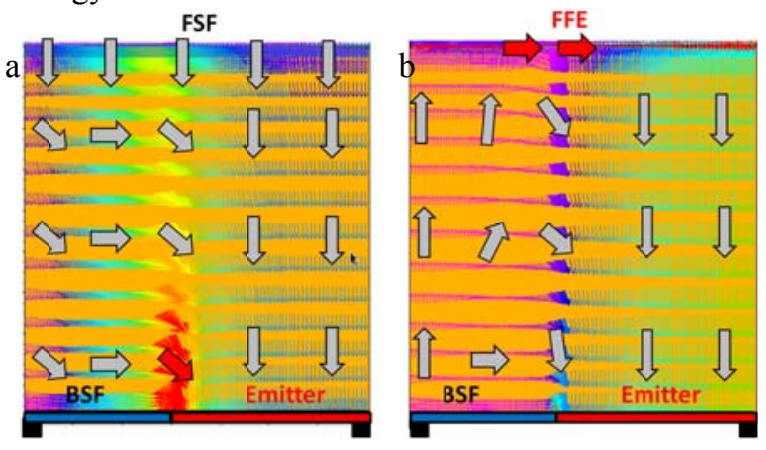

Fig. 2. Current vector plots from 2D numerical device simulations (Atlas), showing the hole current flow over the cross-section of an IBC cell either with (a) FSF or (b) FFE. The big arrows indicate the overall current flow direction and the red arrows indicate the largest current densities.

Fig. 2 compares the flow of holes in a unit cell consisting of $1 / 2$ a BSF and $1 / 2$ an emitter for both the FSF and FFE case. In the FSF case the photogenerated holes in the base over the BSF need to be collected laterally by the nearest emitter. The transport requires a concentration gradient in the holes, and 
hence the carrier concentrations above the BSF become high when the BSF is wide, leading to recombination. In the FFE case the hole current flow is completely different. The photogenerated holes over the BSF are collected in the FFE, then transported as majority carriers through the FFE to the FFE above the rear emitter, where they are re-injected for subsequent collection by the rear emitter. Effectively the holes are pumped from the BSF region to the emitter contact.

The pumping effect in this way increases the transport length for holes generated above the BSF, allowing the BSF width to be as wide as the emitter width without significant loss in cell efficiency. With proper tuning, the FFE can be applied as an effective means to increase the BSF width with marginal loss in cell performance while assuring proc ess simplification and cost reduction. Besides this, the design leads to more freedom in the interconnection lay-out and increases the tolerances for the module fabrication.

\section{VOLTAGE MAPS OF THE FFE AND PUMPING}

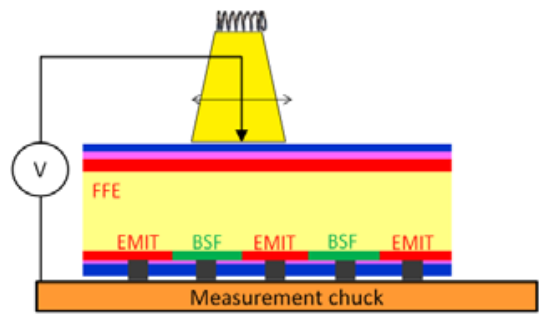

Fig. 3. Mapping the FFE voltage relative to the rear side metallization with the corescan.

Fig. 3 shows how the Sunlab ${ }^{\circledR}$ corescan instrument [7] can be used to map the FFE voltage. The IBC cell is mounted on the chuck, the BSF and emitter contacts are shorted by the chuck. The instrument features a white light source with a beam diameter of about $1 \mathrm{~cm}$. In the center of the beam there is a voltage probe, sensing the FFE voltage relative to the chuck. The light beam together with the voltage probe is scanned across the wafer, resulting in a map of the FFE voltage. When evaluating the maps we need to keep in mind that because of the local illumination used in the FFE voltage map, holes might leak laterally to outside of the spot, leading to less charging of the FFE, resulting in a lower FFE voltage than would occur in a cell operating in full area illumination.

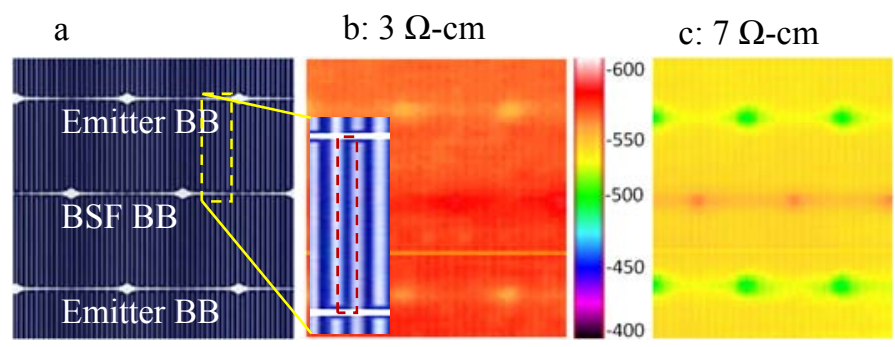

Fig. 4. (a) optical picture of rear side of cell, and (b) and (c) voltage scans $(\mathrm{mV})$ of the FFE at the front side. The horizontal yellow line is an artefact.

The IBC cells feature an interdigitated pattern on the rear side, as shown in Fig. 4a. The BSF and emitter fingers are connected to the BSF and emitter busbars respectively. The busbars for both polarities conduct the current to contact pads. The cells in this case feature a $1 \mathrm{~mm}$ wide BSF, as well as a 1 $\mathrm{mm}$ wide emitter. The contact pads allow for measuring the cell on a chuck. More importantly they allow for easy interconnection and encapsulation of IBC cells in a module. The pads serve to mount the cell on a conductive patterned foil for instance with conductive adhesives [8].

Fig. $4 \mathrm{~b}$ and Fig. $4 \mathrm{c}$ show examples of voltage maps of the FFE. The voltage of the FFE was sensed relative to the rear side contacts, while the cell was illuminated and kept in shortcircuit conditions. In the p-type FFE the majority carriers holes flow from high to low voltage: $J_{h}=\nabla V_{F F E}$.

Despite the metallization being at the rear side of the cell, the features in FFE voltage maps at the front side of IBC FFE cells are clearly correlated with the rear side metallization of the cell.

- The BSF busbars are visible as areas of higher voltage. In particular the wider pads of the busbars are well recognizable. There is a net hole current flow away from above the busbar and its pads. The source of this current is the collection of holes by the FFE from the base.

- The emitter busbars are well visible as well, however as areas of lower voltage, in particular above the wider pad areas: There is a net hole current flow towards the busbar and its pads. The sink of this current is the injection of holes from the FFE into the base and their subsequent collection by the rear emitter. The effects of the pads on the FFE voltage is noticeable over significant distances, certainly larger than the pad diameter.

- The finger pattern is clearly visible, with the voltage higher above the BSF fingers, lower above the emitter fingers. This implies that current is flowing through the FFE from above the BSF to above the emitter, providing direct evidence of the pumping effect in progress.

\section{MERCURY CELLS AND THEIR EQUIVALENT CIRCUIT}

Fig. 5 shows the type of equivalent circuit we used, superimposed upon a schematic of the FFE IBC cell. The pink areas denote the p-type doped (emitter and FFE) regions, the blue areas the n-type doped (base and BSF) regions. 
$43^{\text {rd }}$ IEEE Photovoltaic Specialists Conference, June 5-10, 2016, Portland, USA

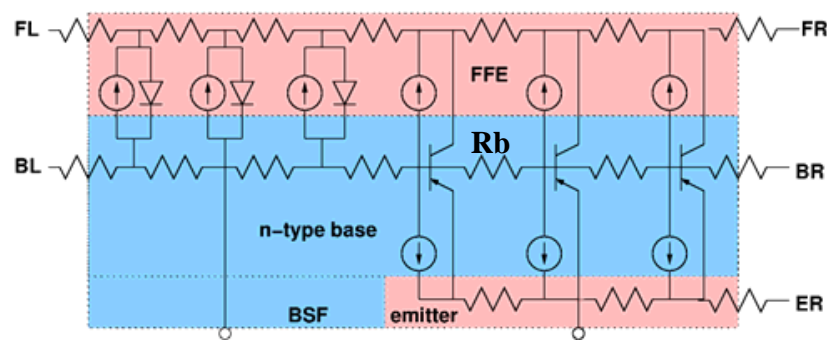

Fig. 5. sample equivalent circuit used for unit cell.

The wires and resistors represent majority carrier transport, electrons in base and BSF, holes in FFE and rear emitter. Minority carrier transport and junctions are modelled within non-linear diode and transistor circuit elements. The illuminated diodes represent the base-FFE junction above the $\mathrm{BSF}$, as in a normal p-n junction solar cell. The illuminated transistors represent the emitter-base-FFE parts of the IBC cell. Equivalent circuits for FFE type back contacted cells employing transistors have been proposed before in for instance $[5,12]$

For the FFE-base-emitter to operate as a transistor, the requirement is that the minority carriers must be able to cross the base, e.g. their diffusion length $\mathrm{L}_{\mathrm{h}}$ must be larger than the thickness $\mathrm{w}$ of the wafer. This condition must be satisfied in any IBC cell, because carriers generated at the front side must be able to diffuse to the junction at the rear side. If not, the current of the IBC cell would be unacceptably low. In for instance an Ebers-Moll model for the transistor this is achieved with a high transistor gain.

Units such as these can be connected together using the nodes FL, FR, BL, BR and ER. Here $\mathrm{L}$ and $\mathrm{R}$ are an abbreviation for left and right, F, B, E for FFE, base and emitter respectively.

In literature, circuit simulation to model a cell beyond the basic unit cell has been described before, for instance [10, 11]. What these approaches have in common is that device simulation is used to simulate different representative parts of the cell. The results of these device simulations are summarized as a 2-diode equivalent circuit. This is a 2terminal circuit. This approach is justified if there are no significant current flows into or out of the unit cell in addition to the current flows through the contacts. In the case of the mercury IBC cell the FFE allows current flows of the same order of magnitude as through the contacts over relatively large distances. A two terminal equivalent circuit is therefore usually not good enough in our case.

\section{CALIBRATION OF CIRCUIT SIMULATION VS QUOKKA}

An important aspect turns out to be the base resistivity of the wafer, in particular over the rear emitter (e.g. resistor $\mathrm{Rb}$ in Fig. 5. In that region lateral transport of $\mathrm{e}^{-}$towards the nearest BSF is occurring. The associated series resistance loss has a big impact on the fill factor, more so as the emitter becomes wider, and bulk resistivity becomes higher. When the resistor values in the base are calculated from the bulk resistivity only, the fill factors produced by the circuit simulation are too high.

For efficient IBC cells however the injection level in the base is quite high, also well below $\mathrm{V}_{\text {oc }}$. The bulk resistivity needs to be reduced for the increased carrier densities. Since the injection level varies with the operating voltage, the resistor values become non-linear and will depend on the voltages in the simulation. The emitter voltage $\mathrm{V}_{\mathrm{emi}}$ and base voltage $\mathrm{V}_{\text {base }}$ are linked to the excess carrier concentration $\Delta \mathrm{p}$ :

$$
V_{\text {emi }}-V_{\text {base }}=\frac{k T}{q} \log \left(\frac{n p}{n_{i}{ }^{2}}\right)=V_{T} \log \left(\frac{\left(N_{D}+\Delta p\right) \Delta p}{n_{i}{ }^{2}}\right)
$$

Solving this equation the injection level $\Delta \mathrm{p}$ can be extracted from the node voltages:

$$
\Delta p=N_{d}\left(\frac{1}{2}-\sqrt{\frac{1}{4}+\left(\frac{n_{i}}{N_{D}}\right)^{2} \exp \left(\frac{V_{e m i}-V_{\text {base }}}{V_{T}}\right)}\right)
$$

From $\mathrm{N}_{\mathrm{d}}$ and $\Delta \mathrm{p}$ and the mobilities the total base conductivity can be retrieved.
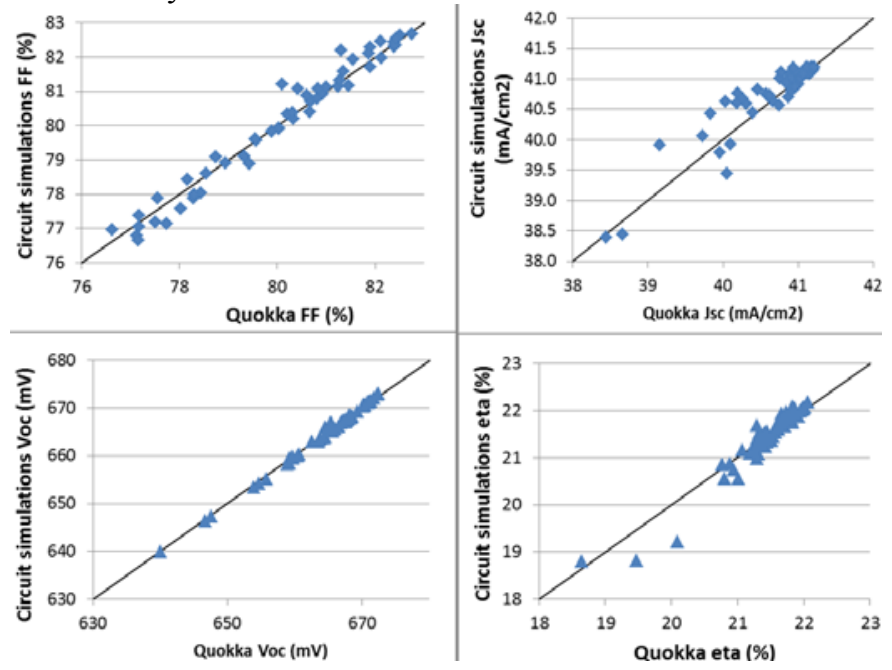

Fig. 6. comparison of circuit simulation I-V parameters vs quokka device simulation parameters, for unit cell as specified in Fig. 4 b).

We implemented such a high-level-injection correction of the bulk resistivity, after application of which we obtain the result in Fig. 6. 


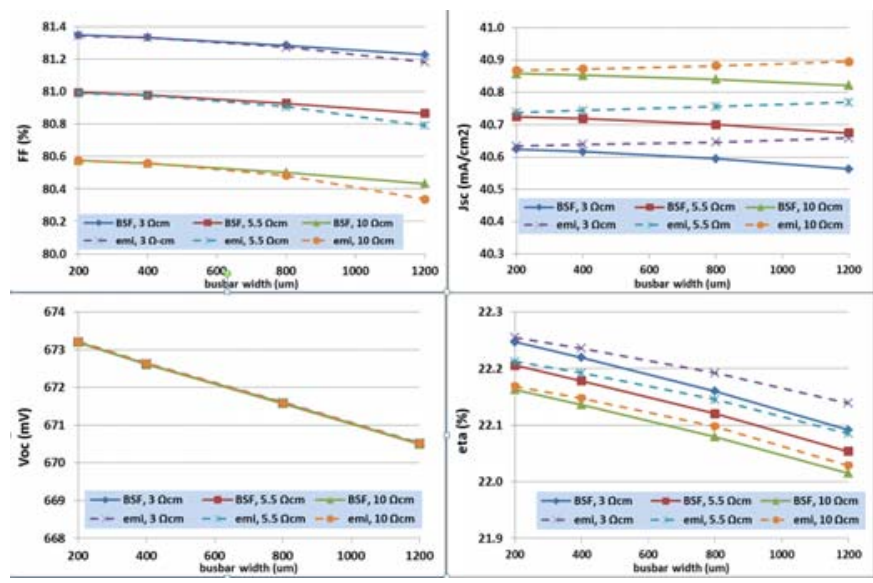

Fig. 7. Simulation results for busbar widths

In Fig. 6 we show a comparison of Quokka device simulation results vs circuit simulation results for a unit cell simulation. Each data point represents a unit cell with a different BSF width, emitter width, bulk resistivity. The emitter- and BSF width ranged from $200 \mathrm{um}$ to $2 \mathrm{~mm}$, the bulk resistivity ranged from 3 to $10 \Omega-\mathrm{cm}$. We can see that over a wide range consistency is observed.

In Fig. 7 we show application of the circuit model in a busbar width study. The area studied is the dashed - orange rectangle indicated in Fig. 4. It contains $1 / 2$ a BSF, $1 / 2$ an emitter and $1 / 2$ a busbar. Since we have 8 busbars in our design for a 6 " cell, the finger length is about $1.95 \mathrm{~cm}$. The BSF and emitter width were fixed at $1 \mathrm{~mm}$, the busbar width was varied. In Fig. 7 we show the I-V parameters, as function of busbar width, for 3 bulk resistivities $(3,5.5,10 \Omega$-cm), for both emitter and BSF busbars.

We can see that emitter and BSF busbars have different impact on the cell parameters. Wide emitter busbars promote the pumping effect due to larger voltage gradients in the base, and result in enhanced current collection, but at the expense of a lower FF. Wide BSF busbars result in some electrical shading. Both busbars result in a voltage drop with increasing width due to the increased metal coverage. The efficiency losses to both types of busbar are in the end quite similar, and independent of resistivity: $0.15 \%$ absolute is lost for a $1.2 \mathrm{~mm}$ busbar. The losses increase with busbar width. In our cell design we typically use narrower and tapered busbars. This type of analysis allows us to design busbars, taking into account not only ohmic losses in the busbars, but also FF, $\mathrm{J}_{\mathrm{sc}}$ and $V_{o c}$ effects due to lateral hole and electron transport effects specific to IBC cells.

\section{CONCLUSIONS AND OUTLOOK}

The FFE voltage scan is an easy to use and telling diagnostic for the FFE IBC cell. The resulting voltage map can well be linked to the cell geometry. The current flow through the FFE can be derived from the voltage map.
Equivalent circuit techniques provide an effective means to model effects beyond unit cells and reveal the physics at work at the bus bars, and explain FF and current effects. The lateral currents in the base and FFE are associated with voltage gradients in FFE and base. They have the effect of moving the base-FFE junction towards higher forward bias. Over the rear emitter this has the effect of enhancing the re-injection of holes into the base, enhancing the pumping effect. Over the BSF this has the effect of less effective carrier collection into the FFE, resulting in electrical shading.

For the circuit simulation we calculate the base resistor values in dependence of the injection level. The circuit simulation has been calibrated against 2-D quokka simulations for the cell case. The circuit simulation has then been used as a tool to work out the impact of both BSF and emitter busbars on cell performance.

The authors gratefully acknowledge the support of the Dutch Ministry of Economic Affairs, within the TKI framework, projects IBChampion and IBCense.

\section{References}

[1] Smith et al. "SunPower's Maxeon Gen III solar cell: High Efficiency and Energy Yield", Proc. $39^{\text {th }}$ IEEE PVSC, Tampa, 2013.

[2] M. Hermle, et al. "Shading Effects in Back-Junction BackContacted Silicon Solar Cells", Proc. 33 ${ }^{\text {rd }}$ IEEE Photovoltaic Specialists Conference, San Diego, CA, 2008.

[3] I. Cesar I, N. Guillevin N, A.R. Burgers, A. A. Mewe, M. Koppes, J. Anker, L. J. Geerligs, A. W. Weeber, "Mercury: A back junction back contact front floating emitter cell with novel design for high efficiency and simplified processing", Energy Procedia.2014;55:633 - 42.

[4] R. Gutiérrez, F. Hernando, J. C. Jimeno, S. Uriarte, "Industrial manufacturing process of silicon TWT solar cell", Proc. $19^{\text {th }}$ EU PVSEC, Paris, 2004.

[5] C. Sah et Al. "Floating Emitter Solar Cell", US patent 4,665,277, 1987.

[6] I.G. Romijn et Al. "Industrial Cost Effective N-Pasha Solar Cells with $>20 \%$ Cell Efficiency", Proc. $29^{\text {th }}$ EU PVSEC, Amsterdam, 2014

[7] A. S. H. van der Heide, J. H. Bultman, J. Hoornstra, A. Schönecker," Error diagnosis and optimisation of C-Si solar cell processing using contact resistances determined with the Corescanner", Solar Energy Materials \& Solar Cells, 2002;43:p.43-.

[8] P. C. de Jong et Al. "Progress made with back-contact modules using conductive adhesive interconnection technology", Proc. $22^{\text {nd }}$ EU PVSEC, Milano, 2007.

[9] J. J. Ebers, J. L. Moll, "Large-signal behavior of junction transistors", Proceedings of the Institute of Radio Engineers, 1954;42:1761-1772.

[10]P. Magnone P, M. Debucquoy, D. Giaffreda, N. Posthuma, C. Fiegna, "Understanding the influence of busbars in large-area IBC solar cells by distributed SPICE Simulations", IEEE J. Phot, 2015;5:552-8.

[11]A. Fell, K. C. Fong, K. R. McIntosh, E. Franklin, A. W. Blakers, "3-D simulation of Interdigitated-Back-Contact silicon solar cells with Quokka including perimeter losses", IEEE J. Phot, 2014;4:1040-4. 
$43^{\text {rd }}$ IEEE Photovoltaic Specialists Conference, June 5-10, 2016, Portland, USA

[12]J. C. Jimeno, S. Uriarte, C. Ikaran, R. Nafarrate, N. Barbazán, I. Petrina, "Modeling the TWT structure", Proc. $19^{\text {th }}$ EU PVSEC,

Paris, 2004. 